\title{
The Microarray Data Analysis Process: From Raw Data to Biological Significance
}

\author{
N. Eric Olson \\ VizX Labs, Seattle, Washington
}

\begin{abstract}
Summary: Despite advances in microarray technology that have led to increased reproducibility and substantial reductions in the cost of microarrays, the successful use of this technology is still elusive for many researchers, and microarray data analysis in particular presents a substantial bottleneck for many biomedical researchers. There are many reasons for this, including the expense of and a lack of adequate training in the use of analysis software. An additional reason is that microarray data analysis has largely been treated in the past as a set of separate steps, with the majority of emphasis being placed on statistical analysis and visualization of the data. For many biomedical researchers determining the biological significance of the data has been the greatest challenge and in the last several years more emphasis has been placed on this aspect of
\end{abstract}

the analysis process. Despite this broadening of the scope of analysis there are still several aspects of the process that continue to be neglected, including additional related and interdependent aspects, such as experimental design, data accessibility, and platform selection. Though not traditionally thought of as integral to the data analysis process, these factors have profound effects on the analysis process. This article will discuss the importance of these additional aspects, as well as statistical analysis and determination of biological significance of microarray data. A summary of currently available software options will also be presented with a focus on the aspects discussed. Key Words: Microarray data analysis, biological significance, microarray, statistical analysis, clustering.

\section{INTRODUCTION}

Microarrays allow biological researchers to examine the mRNA expression levels of tens of thousands of genes at once. This advance has given biomedical researchers the opportunity to examine expression for an entire genome, where previously they could only look at a handful of genes simultaneously. (The reader is referred to Miller and Federoff ${ }^{1}$ in this issue for an excellent review of the use of microarrays in the study of Parkinson's disease.) Initially, factors such as low data quality and expense limited the usefulness of this technology for all but a few researchers. However, improvements in the hardware and related technology have not only increased the quality and reproducibility of data, but also made the arrays themselves less expensive, making microarray research accessible to most biomedical researchers.

Despite these advances in microarray technologies, bottlenecks still exist in microarray experiments for many researchers, and the analysis of the large amounts

Address correspondence and reprint requests to: N. Eric Olson, Ph.D., VizX Labs, 200 West Mercer Street, Suite 500, Seattle WA, 98119. E-mail: eric@vizxlabs.com. of data produced by such research remains a significant challenge. There are many reasons for this, including a lack of adequate training in the use of analysis software adding to the already high cost of the software itself. An additional reason is that microarray data analysis has largely been treated in the past as a set of separate steps, and microarray users often found themselves using multiple software packages to navigate their way through the analysis process. An additional problem was that the most emphasis was being placed on statistical analysis and visualization of the data, while for most biomedical researchers using microarrays, determining the biological significance of the data has been the greatest challenge, and in the last several years more emphasis has been placed on this aspect of the analysis process. Despite this broadening of the scope of the analysis process, there are still several aspects of the process and their relationship to analysis software that continue to be neglected. In reality, the process can be expanded to include additional related and interdependent aspects, such as experimental design, data management, accessibility, and platform selection, that are not traditionally thought of as integral to the data analysis process but that nonetheless can have profound effects on statistical analysis. 


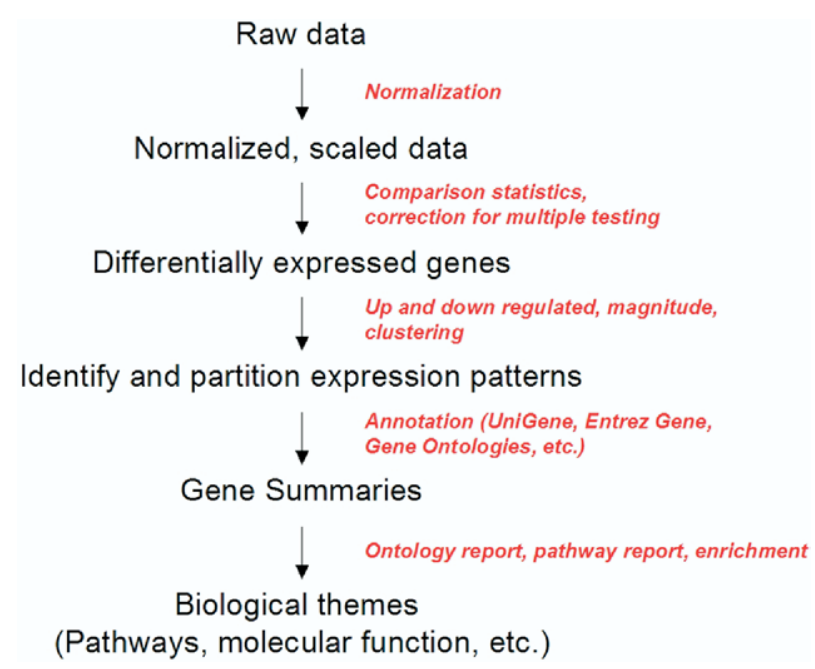

FIG. 1. The microarray data analysis process. The overall goal of the microarray data analysis process is to take raw expression data and identify the biological significance of this data. While this general workflow applies to most experiments, the specific methods used at each step will depend on factors such as experimental design and the array platform being used.

The overview presented here will discuss the importance of these additional aspects as well as statistical analysis and determination of biological significance and emphasize how each aspect affects the analysis process as a whole. A summary of currently available software options, with an emphasis on commercial software systems, will also be presented with discussion of how each addresses the different aspects of the analysis process.

\section{THE MICROARRAY DATA ANALYSIS PROCESS}

The types of microarray studies can be divided into three general groups based on the goal: class comparison, class discovery, and class prediction. ${ }^{2}$ For class comparisons the goal is to identify genes that are differentially expressed between two or more groups. The groups can represent different biological states such as disease state, histologic subtype, or treatment group. For class discovery studies the goal is to identify groups with the samples examined or within the genes examined. Class prediction studies involve trying to predict group membership for a sample based on gene expression profiles. This review will focus on class comparison and class discovery studies and will discuss experimental design and statistical analysis relative to these types of studies.

Converting the data provided in a microarray dataset into meaningful biological knowledge still remains the primary challenge for many researchers. Figure 1 gives an overview of the steps involved in moving from raw data to biological significance. The key steps required for this conversion can be broken down into three discrete sets of tasks: identification of significantly regulated genes, identification of global patterns of gene expression, and the determination of the biological meaning of both individual genes and groups of genes. If experiments are performed with an adequate number of replicates, there are several standard comparative statistical tests available that will generate a list of genes whose differential expression is statistically significant. ${ }^{3}$ Depending on the design of the experiment, for the second step, unsupervised clustering methods can then be used to identify patterns of gene expression among the differentially regulated genes. Although these two steps are critical in the evaluation of microarray data, the third step of determining the biological significance of the genes identified is perhaps the most important in the analysis of these types of data. This analysis is often done on a gene-by-gene basis using annotation supplied by genomic databases. Several challenges exist with this approach. First, a reliable source of gene annotation must be available. If the analysis is performed manually, this can be extremely time consuming unless the number of genes in the gene list is somehow limited. However, analyzing only a small subset of the data can lose the larger biological context. Automation of microarray data analysis promises to overcome these difficulties and provide high-quality, meaningful information in a short period of time.

\section{EXPERIMENTAL DESIGN}

Careful experimental design is essential to the successful use of microarrays because it determines what can be inferred from the data as well as the confidence that can be assigned to those inferences. Two of the most important aspects of experimental design are the type of experiment and the use of replicates.

\section{Design of experiment}

The type of experiment will affect the downstream analysis of the data. A comparison between two conditions, for example, control and experimental, will require a twogroup test such as a $t$ test. A multiple condition experiment (such as a time course where several time points are being compared with control) requires a test that can be used for more than two samples such as analysis of variance (ANOVA). The type of ANOVA that will be needed will depend on the experimental design, in particular how many factors are being examined. Examples of factors that might be examined in typical microarray experiments include disease state, strain of model organism, drug treatment, or time after treatment. If one factor is being studied, for example, time after treatment in a time series, a one-way ANOVA may be used. If multiple factors are being studied, then a two-way ANOVA is needed. ${ }^{4}$ An example of a two-factor study would be one that looks at response to treatment (e.g., a neurotherapeutics) in two different strains of mice. In this 
type of study we are interested in identifying three types of genes: those that differ between strains, those that are regulated by treatment, and those in which there is interaction between the strain in treatment; e.g., genes that are regulated by treatment in only one strain. The type of experiment, number of groups, and number of factors examined will affect the choice of statistical methods used to detect differential expression.

\section{Replicates}

The use of replicates aids in distinguishing between genes that are truly differentially expressed and those affected only by noise. Averaging over replicates minimizes the effects of chance variation and allows the extent of experimental variation to be estimated. Comparison tests use this estimate of variability within the replicates to assign a confidence level as to whether the gene is differentially expressed. Two types of replicates are generally considered for microarray experiments: technical and biological. Technical replicates deal with technical variation and an example would be hybridizing the same sample to three different arrays. Biological replicates are used to deal with biological variability that may be due to genetic variability or environmental effects. An example of biological replicates would be extracting RNA from brains from three different mice and hybridizing each sample to its own array. Three replicates should be considered a minimum, but studies suggest that four or five replicates would be a better minimum. ${ }^{5}$ The exact number of replicates required depends on the variability inherent in the system being studied, with studies using patient data usually requiring more than studies using animal models or cell lines. Regardless of the system being studied, it is important to realize that rigorous statistical inferences cannot be made with a sample size of one and, in general, the more replicates, the stronger the inferences that can be made from the data.

\section{PLATFORM SELECTION AND COMPATIBILITY}

Many different microarray platforms exist, including spotted cDNA, spotted oligo microarrays, and in situ synthesized oligo microarrays. In addition to the type of probe used, platforms also vary between one or two color platforms. The choice of platforms will have a substantial effect on the data analysis process. Two color arrays have several options for how the experiment can be designed. The second channel can either be part of the experiment (i.e., a control RNA) or it can be an external reference RNA that is strictly used for the creation of a ratio. For single-channel arrays the sample is hybridized to one array and the signal intensity is used for measuring gene expression. Although both one- and two-color platforms can be used successfully, experimental design and analysis is generally more straightforward with onecolor microarrays.

Another important consideration for data analysis is the platform's data format. Each platform makes the data available in a particular format and it is important to make sure that the software used for analysis can easily accommodate the array platform being used in terms of loading the data for analysis and normalizing the data in the appropriate way and that it provides access to the analysis tools that may be required for the particular experimental layout used for that platform. Many software systems are designed either for use with one particular array platform or the system is optimized for a specific platform, making analysis of other array platforms much more difficult. The use of all of the common commercial platforms is well supported in the literature and, despite earlier studies reporting poor agreement in cross-platform comparisons, recent studies have shown very good agreement between different array platforms. ${ }^{6-9}$ This improved concordance between platforms can be attributed to general improvements in microarray technology and a more comprehensive experimental design in the recent studies; the studies cited generally included more replicates than the earlier studies. The choice of microarray platform can therefore be driven primarily by factors such as availability of a reliable core facility, ease of experimental design, and availability of analysis software for the platform.

\section{DIFFERENTIAL EXPRESSION}

The fundamental goal of microarray expression experiments is to identify genes that are differentially expressed in the conditions being studied. Additionally, the identification of discrete patterns of gene expression and subsequent partitioning of the data set based on expression patterns is commonly done after the identification of differentially expressed genes. Therefore the analysis process can typically be broken down into two steps: (1) filtering to identify those differentially expressed genes using a combination of criteria, including fold change and statistical significance determined by comparison statistics, and (2) separation of those differentially expressed genes into discrete groups or clusters based on expression pattern. Depending on the experimental design this second step may be a simple as generating a list of genes with increased expression or decreased expression or it may involve the use of cluster analysis to identify more complex patterns of gene expression.

Factors such as quality control of the raw data and normalization are also relevant to data analysis. Without good quality data, reliable identification of differentially expressed genes is not possible. Initial quality metrics are usually generated by the software used to generate the 
raw data from the array images. This software is often provided by the array manufacturer and researchers should follow the manufacturer's recommendations regarding acceptable values for quality parameters. This is not to say that researchers are limited to the quality metrics provide by array manufacturers for quality assessment; there are additional tools available for quality assessment for specific platforms and the reader is referred to a recent paper by Reimers et al ${ }^{10}$ that provides a review of quality assessment for the Affymetrix platform. With most commercial array platforms, normalization is often performed as part of the initial data extraction process and the majority of biomedical researchers will, at least initially, start with prenormalized data. Additionally, like quality control metrics, normalization methods are often platform dependent. Because of these two factors, the differences in normalization methods for specific platforms will not be discussed in this review, beyond making the statement that, although there is considerable debate about the benefits of various methods, the use of all methods supported by the major commercial microarrays is well supported in the literature. There are several good reviews discussing and comparing the different normalization options for specific platforms and the reader is directed to these references. ${ }^{11-14}$

\section{Comparison statistics}

The first issue that arises in identifying those genes with differential expression is "How confident are we that the changes in expression we find are real?" When selecting genes showing differential expression, it is necessary to establish criteria to be used in determining whether a gene shows altered expression between two or more different conditions. (The reader is referred to Almudevar and coworkers ${ }^{15}$ in this issue for an excellent discussion on using correlation measures in the analysis of gene expression data.) The criteria used will influence the content of the list and determine how confident we are that the genes identified are really differentially regulated. Although it has been commonly used, the simple use of fold change does not address the reproducibility of the observed difference and therefore cannot be used to determine the statistical significance of changes in expression. For this comparison statistics are needed; these tests require replicates and use the variability within the replicates to assign a confidence level or $p$ value as to whether the gene is differentially expressed. The commonly used comparison tests can be grouped based on the number of groups being compared as well as the number of factors being examined. The $t$ test, Welch's $t$ test, and the Wilcoxon Rank Sum can be used for twogroup comparisons. If three or more groups are being compared, then one-way ANOVA or the Kruskal-Wallis test can be used if only one factor is being examined. If two or more factors are being examined, then two-way ANOVA should be used.

The comparison tests can also be grouped based on how they deal with differences between groups and variability within groups. Parametric tests, like the $t$ test, identify genes where there is a large difference between the groups and a small amount of variability within the groups, that is, where the values for the replicates in each group are similar. Nonparametric tests, like the Wilcoxon rank sum test, identify genes where all or most of the values for the replicates in one group are higher than values in the other group. These tests do not assess the variability between replicates in a group, they only determine whether the values in one group are higher or lower than most of the values in the other group. A parametric test, such as the $t$ test, is especially useful for experiments using model systems such as animal models or cell lines, in which the researcher expects good agreement between replicates and excessive variability between replicates indicates either technical artifacts or nonuniform biological response across replicates. Nonparametric tests, such as the Wilcoxon rank sum test, can be useful when data from patients are being examined and there may be a large amount of variability between replicates because of factors such as differences in sex and age between patients. In addition to these standard statistical tests, there are also variations on these tests that have been developed specifically for use with microarray data that gain statistical power for studies with limited replicates by calculating variance from a pool of genes with similar expression levels rather than using only the values for the gene being measured. ${ }^{16}$

\section{Correction for multiple testing}

Once a $p$ value has been calculated for each individual gene being examined, that $p$ value should then be adjusted based on the number of tests performed. The reason for this recommendation is that the $p$ value calculated for an individual gene represents the chance of a similar difference occurring due to chance and this risk is cumulative for all tests being performed. That is, if 10,000 tests are performed and we use a 0.05 cutoff for the raw $p$ value, we would expect 500 false positives. Multiple testing corrections allow the user to adjust the $p$ value based on the number of tests performed. ${ }^{17-19}$ These adjustments help to reduce the number of false positives in an experiment. Family wise error rate (FWER) corrections adjust the $p$ value so that it reflects the chance of at least one false positive being found in the list. Therefore, if we identified 500 genes with an adjusted FWER $p$ value of 0.05 , then there is a $5 \%$ chance of having 1 false positive in the list of 500. Examples of FWER methods include Bonferonni and Holm. False discovery rate corrections (FDR) adjust the $p$ value so that it reflects the frequency of false positives in the list. There- 

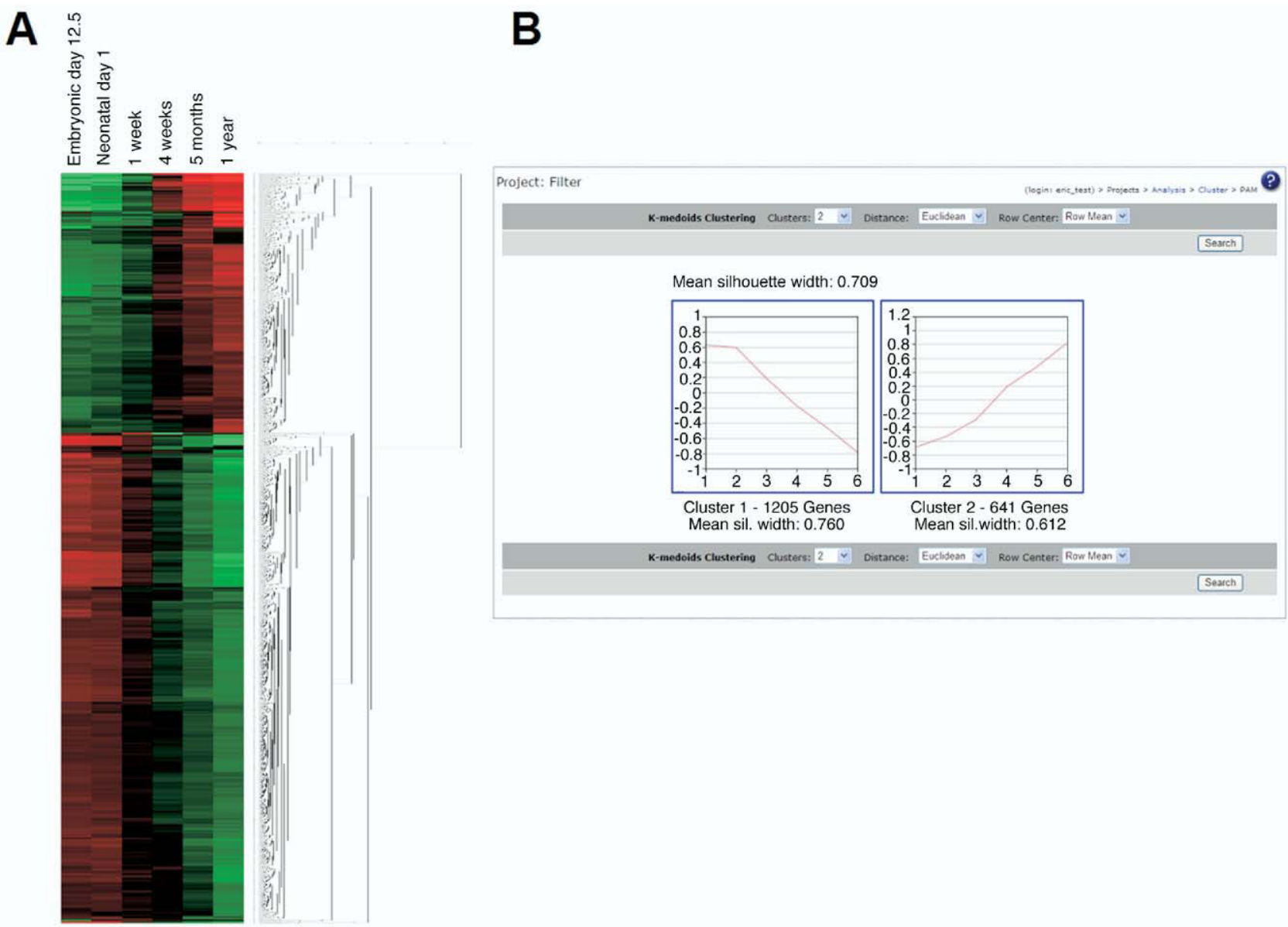

FIG. 2. Clustering of 1846 differentially expressed genes from FVB heart development time series. Two different clustering methods were applied to the same set of 1846 genes. (A) Hierarchical clustering of 1846 differentially expressed genes. Hierarchical clustering sorts and groups the genes so that genes with similar expression profiles are placed close together, making it easier to identify global patterns of gene expression. The heatmap on the left represents the expression level for each sample, with green signifying low expression and red high expression. (B) The same 1846 genes were clustered using a partitioning method (Partitioning Around Medoids) into two clusters.

fore, if we identified 500 genes with an adjusted FDR $p$ value of 0.05 , then there are an estimated 25 false positive in the list of 500. Examples of FDR methods include Benjamini and Hochberg and SAM. The FWER methods are more conservative, but the FDR methods are usually acceptable for "discovery" experiments; i.e., where a small number of false positives is acceptable.

\section{Identification of expression patterns}

After we have identified a list of genes showing differential expression we may want to separate those genes based on expression pattern. This may simply consist of separating genes into those that show increased expression and those that show decreased expression if we are examining two conditions. For more complex experimental designs, such as time series, we have the potential for many different patterns of gene expression based not only on direction of change but also time of changes. The researcher may want to split the set of differentially expressed genes into many different discrete groups or cluster patterns of gene expression using cluster analysis. Clustering methods are descriptive or exploratory tools that can be used to identify groups within complex datasets. ${ }^{20-22}$ Clustering methods can be used to identify patterns of gene expression in microarray datasets. Methods such as hierarchical clustering can be used to help identify patterns in a large dataset, while partitioning methods such as $K$ means clustering can be used to separate data into discrete groups or clusters. Hierarchical methods provide a hierarchy of clusters, from the smallest, where all objects (gene expression profiles) are in one cluster, through to the largest set, where each observation is in its own cluster. Partitioning methods partition the data (list of genes) into a prespecified number $(K)$ of mutually exclusive groups based on a feature vector (expression profile). Figure 2 shows both types of clustering applied to the same set of differentially expressed genes. Using hierarchical clustering for visualization, two global patterns of gene expression are 
readily identifiable. Partition clustering is then used to split the list of differentially expressed genes into two separate lists based on these global expression patterns.

\section{BIOLOGICAL SIGNIFICANCE}

Once a list of differentially expressed genes has been generated, the next task to determine the biological significance of the genes in that list. There are a variety of public databases available that provide different types of information for genes and gene products. By combining the identification of broad biological themes with the ability to focus in on a particular gene, it is possible to rapidly characterize the biology involved in a particular experiment and to identify particular genes of interest from a very large list of potential targets. A short description of some of the most commonly used gene annotation sources is included below. ${ }^{23}$

\section{GENE ANNOTATION SOURCES}

Genbank (http://www.ncbi.nlm.nih.gov/Genbank/). Sequence information for particular clones and probes.

UniGene(http://www.ncbi.nlm.nih.gov/entrez/ query.fcgi?db=unigene). Organizes GenBank sequences into a nonredundant set of gene-oriented clusters. Gene titles are assigned to the clusters and these titles are commonly used in microarray research to refer to that particular gene.

Entrez Gene (LocusLink) (http://www.ncbi.nlm. nih.gov/entrez/query.fcgi?db=gene). Provides a single-query interface to curated sequence and descriptive information, including function, about genes.

Ensembl Genome Browser (http://www.ensembl. org/). Produces and maintains automatic annotation on selected eukaryotic genomes, concentrating on vertebrate genomes.

Gene Ontology (http://geneontology.org/). The Gene Ontology Consortium provides controlled vocabularies for the description of the molecular function, biological process, and cellular component of gene products, that can be used by databases such as Entrez Gene.

KEGG Pathways (http://www.genome.jp/kegg/ pathway.html). Kyoto Encyclopedia of Genes and Genomes provides information about both regulatory and metabolic pathways for genes.

OMIM (http://www.ncbi.nlm.nih.gov/entrez/query. fcgi?db=OMIM). A catalog of human genes and genetic disorders.

HomoloGene (http://www.ncbi.nlm.nih.gov/entrez/ query.fcgi?db=homologene). A gene homology tool that compares nucleotide sequences between pairs of organisms, providing automated detection of homologs among the annotated genes of several eukaryotic ge- nomes. Using HomoloGene, annotation can be combined and compared across organisms.

Information from annotation sources can be used to identify individual targets for further study, to prioritize the genes on the list, or to identify broad biological themes in the entire list. Using these sources of annotation, users can examine individual genes in the list, including information about function and role in disease. This information can be used to identify interesting targets for further study or to prioritize the genes on the list. For example, a researcher may focus on a particular gene or group of genes because of their involvement in a particular disease or involvement in a particular biological process. The gene list as a whole also provides information about the experiment. By examining the entire list, broad biological themes may be identified. A particular treatment may affect the expression of particular types of genes. For example, during a differentiation timecourse, genes involved in the cell cycle may be affected with positive regulators being downregulated as cells differentiate. Rather than examining each differentially expressed gene on a gene-by-gene basis, the functional information such as ontology or pathway terms for the entire list can be summarized. For each term associated with the differentially expressed genes, the report shows how many genes in that list have that ontology and how many on the array have that ontology. A statistical score, such as a $z$ score, can then be assigned, which indicates whether that term is more or less common in the gene list than would be expected by chance. $^{24,25}$ This type of enrichment analysis can rapidly identify specific ontology or pathway terms that are enriched in the list and identify the broad biological themes associated with the gene list.

\section{DATA MANAGEMENT}

Microarray experiments produce tremendous quantities of raw data: depending on the platform and the specific array being used, a single experiment can generate millions of data points. In addition to this raw data, there are large amounts of information about the individual samples, their processing, as well as the hybridization of the arrays that must be managed. Managing the raw data and the associated experimental process annotation is a huge task and an important consideration in the microarray process. Databases can make organizing and retrieving raw data more efficient and can facilitate the storage, management, and retrieval of experimental annotation. Ideally, data can be managed in such a way that retrieval of subsets of the data based on expression values, the results of statistical analysis, sample annotation, or, most importantly, on specific genes or sets of genes can be easily accomplished. Additionally, using a database to create standardized information about sam- 
ples and sample preparation that can aid in keeping track of experimental annotation in a standardized way is also important for meeting requirements for the publication of the data.

\section{DATA AND SYSTEM ACCESSIBILITY}

System access, defined both in terms of physical access and the usability of the microarray data analysis system, is critical for the microarray data analysis process; the people who need to work with the data must be able to access the system when needed in an intuitive way.

Working with large amounts of microarray data requires the use of microarray data analysis software. There are many options available and many of those will be discussed in more detail later in this review, but in terms of system access, microarray data analysis software used in one or many steps of the analysis process falls into three general categories: single-user desktop systems, single-user server-based systems, and webbased systems. Single-user desktop systems are simply computer programs that run on a single computer. While not dependent on networks or servers, these systems are limited to use at one location and one user at a time. Single-user server-based systems are similar to desktop systems, differing in that they can be run over a network and thus can be accessed from multiple computers on a network. Web-based systems run over the Internet or an intranet via a browser. Data encryption and password protection of accounts allow secure access from any computer that can access the network locally or remotely. They may allow sharing data within the same lab and well as with collaborators around the world. These systems are often platform independent, allowing access from any computer with Internet access, regardless of operating system, and have no special hardware requirements for the end user's computer. The type of system used will determine who can access it, how many people can access it, and the locations from which it can be accessed.

Another critical aspect of software that determines accessibility is ease of use and the extent to which specific training is required to use the system. The available software options span a wide spectrum in terms of ease of use and required training, ranging from systems that require extensive bioinformatics expertise and lengthy training to systems that are designed for use by bench researchers with little or no bioinformatics expertise. As all of the analysis methods and tools for determining biological significance covered in this review are available from systems at both ends of this spectrum, the selection of a system can be driven by a researcher's specific needs regarding accessibility, both in terms of physical access and ease of use.

\section{MIAME, MAKING DATA PUBLIC AND USE OF PUBLIC DATA}

When a researcher is ready to publish their microarray work they may be confronted with the Minimum Information About a Microarray Experiment (MIAME) standards and submitting their data to a public database. ${ }^{26,27}$ A subset of the MIAME standard is used as a guideline for submission of microarray materials and methods information in many journals and many journals are now requiring that authors make their data publicly available as a requirement for publication. The MIAME guidelines outline the minimal information that should be included when describing a microarray experiment. The information required includes information such as the array platform, experiment design, and sample preparation. A MIAME checklist is available from the microarray gene expression data (MGED) website. ${ }^{28}$

There are several public repositories for microarray data where users can submit their data and many researchers set up their own website for dissemination of their data. Each database makes data available in a different format. The Gene Expression Omnibus (GEO), maintained by NCBI, is one of the largest and most widely used. ${ }^{29}$ Many researchers are unaware of the many applications the database has for research or do not have an understanding of how to best make use of it. Those who do use the system in their research have a very powerful tool as they seek to better understand gene expression. The GEO offers its own formats as well as making raw data available for many datasets. The GEO currently holds over 60,000 submissions from nearly 1200 researchers, representing over half a billion gene expression measurements for over 100 organisms, drawn mainly from microarray experiments.

This vast collection of microarray data provides researchers with new opportunities and can be used in many ways. For example, it can be used to supplement new experimental data developed by a researcher to provide a control, baseline, or other comparison. Alternatively, data from GEO can be analyzed and explored for significant differential expression patterns previously unobserved, thus allowing researchers to "data mine" the thousands of experiments performed by other laboratories. Public databases are a valuable resource for microarray research, providing thousands of datasets for meta-analysis or re-analysis.

\section{TYPICAL ANALYSIS WORKFLOWS}

Figure 3 presents two typical analysis workflows for common types of experimental design. Although all experiments share some common analysis steps, the exact analysis steps differ and are determined by the experimental design. All three start with a normalized data set 


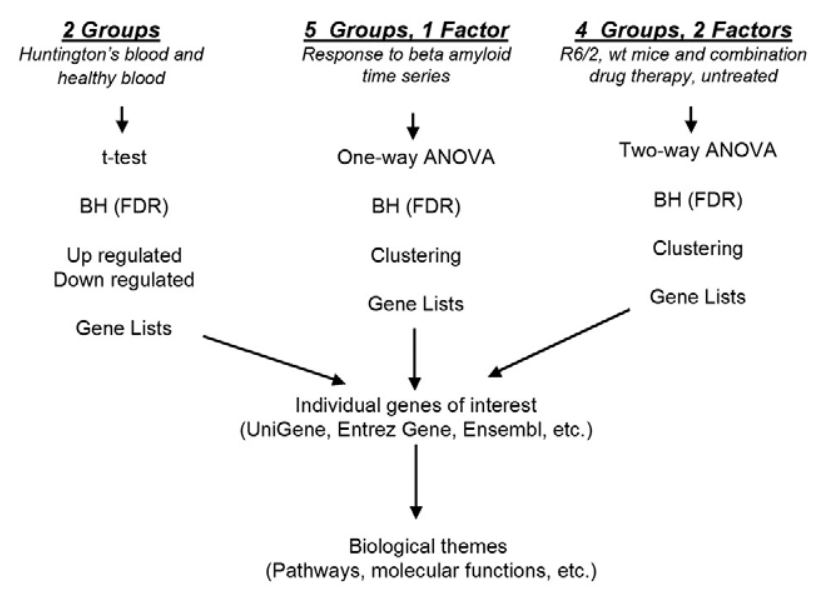

FIG. 3. Typical analysis workflows. Data analysis workflows for three common experimental designs using single-color array platforms. All experiments have at least three biological replicates.

and with the identification of differentially expressed genes, as well as some common filtering elements. The main difference is the type of comparison statistics used. The study comparing gene expression in blood from patients with Huntington's disease with that in blood from healthy donors uses a two-group comparison test (for this example a $t$ test is used) while the time series examining the response to beta amyloid peptide in cultured astrocytes has five groups and requires multiple group comparisons (in this example a one-way ANOVA is used). The first step results in differentially expressed genes that can then be partitioned based on expression pattern. For the two-group comparison this can be done by simply splitting the gene list into two lists, one with genes with increased expression in Huntington's disease and one with decreased expression in Huntington's disease. For this two-group comparison clustering of the groups is not required. However, clustering could be used to look at the similarity of replicates using clustering of individual samples. For the time series there is the potential for more complex patterns of gene expression than simply up- or down-regulation (e.g., there could be patterns showing up-regulation at early time points or late time points as well as upregulation at early times decreasing at later times) and therefore cluster analysis is used to identify more complex patterns of gene expression. For the example shown, hierarchical clustering is used, but principal component analysis could also be helpful in identifying groups within the gene list. Partition clustering can then be used to split the gene list into discrete clusters based on expression pattern so that each cluster can be examined independently.

After gene lists or clusters have been identified and clustered, annotation for each of the individual genes can then be gathered from a variety of sources, allowing examination of the biological significance of individual genes. Functional information such as gene ontology terms and KEGG pathway terms can the be summarized for each of the groups of genes, allowing for the identification of broad biological themes associated with each gene list or cluster.

\section{MICROARRAY DATA ANALYSIS SOFTWARE}

The use of software for microarray data analysis is a necessity. The large volumes of data, the statistical and visualization tools, and the coalescing of disparate annotation sources all require the use of computers. Software for microarray data analysis has been a difficult issue for many biomedical researchers using microarray technology and is still one of the largest challenges to the successful use of microarrays. The types of software available can be broken into two broad categories, commercial software and noncommercial, often public domain, software.

\section{Noncommercial options}

There are many analysis software options that are available free for academics (some of these options may require licensing for commercial use). One of the most widely used by biostatisticians and bioinformaticists is Bioconductor. Although very powerful and flexible, Bioconductor does have a steep learning curve and requires learning the R statistical scripting language. Fortunately many of the features available through Bioconductor are also available through many of the commercial software systems discussed in the next section and many of these systems provide much more accessible interfaces to the Bioconductor package. There are several good reviews of noncommercial options for microarray analysis and the reader is referred to these sources for a more thorough discussion of these options. ${ }^{30-33}$

\section{Commercial software}

Commercial software offers biomedical researchers who lack access to bioinformatics expertise or who simply want to perform the analysis themselves the ability to perform microarray data analysis. Commercial software is typically more stable, better supported and documented, and usually offers an easier-to-use solution. In addition many commercial software solutions offer a higher degree of integration across the multiple steps of the data analysis process; that is the commercial solutions typically cover more of the aspects addressed earlier in this review in one system. Table 1 summarizes the aspects of the analysis process addressed by some of the most commonly used commercial analysis systems. The systems have been divided into three general categories based on the particular aspect of the process the system addresses. More details about each system are provided below for each of the systems. 
TABLE 1.

\begin{tabular}{|c|c|c|c|c|c|c|c|c|}
\hline & $\begin{array}{c}\text { Data } \\
\text { Management }\end{array}$ & $\begin{array}{c}\text { Computer } \\
\text { Platform } \\
\text { Independent }\end{array}$ & $\begin{array}{l}\text { Multiple } \\
\text { Array } \\
\text { Platforms }\end{array}$ & $\begin{array}{c}\text { Web } \\
\text { Accessible }\end{array}$ & $\begin{array}{l}\text { Differential } \\
\text { Expression }\end{array}$ & $\begin{array}{c}\text { Gene } \\
\text { Annotation }\end{array}$ & $\begin{array}{l}\text { Pathway/ } \\
\text { Function } \\
\text { Analysis }\end{array}$ & $\begin{array}{l}\text { Network } \\
\text { Analysis }\end{array}$ \\
\hline Pathway Studio & & & & & & $\mathrm{x}$ & $\mathrm{x}$ & $\mathrm{x}$ \\
\hline Ingenuity & & $\mathrm{x}$ & & $\mathrm{x}$ & & $\mathrm{x}$ & $\mathrm{x}$ & $\mathrm{x}$ \\
\hline PubGene & & $\mathrm{x}$ & $\mathrm{x}$ & $\mathrm{x}$ & & & $\mathrm{x}$ & $\mathrm{x}$ \\
\hline GeneDirector & $\mathrm{x}$ & $\mathrm{X}$ & $\mathrm{x}$ & $\mathrm{x}$ & & & & \\
\hline GeneTraffic & $\mathrm{x}$ & & $\mathrm{x}$ & $\mathrm{x}$ & $\mathrm{x}$ & $\mathrm{x}$ & & \\
\hline Rosetta Resolver & $\mathrm{x}$ & & $\mathrm{x}$ & & $\mathrm{x}$ & $\mathrm{x}$ & & \\
\hline Vector Xpression & & & $\mathrm{x}$ & & $\mathrm{x}$ & $\mathrm{x}$ & & \\
\hline Partek & & & $\mathrm{x}$ & & $\mathrm{x}$ & $\mathrm{x}$ & & \\
\hline GeneSight & & & $\mathrm{x}$ & & $\mathrm{x}$ & $\mathrm{x}$ & $\mathrm{x}$ & \\
\hline GeneSpring & & & $\mathrm{x}$ & & $\mathrm{x}$ & $\mathrm{x}$ & $\mathrm{x}$ & \\
\hline Spotfire & & & $\mathrm{x}$ & & $\mathrm{x}$ & & & \\
\hline ArrayAssist & & & & & $\mathrm{x}$ & $\mathrm{x}$ & & \\
\hline Array Analyzer & & & $\mathrm{x}$ & $\mathrm{x}$ & $\mathrm{x}$ & $\mathrm{x}$ & $\mathrm{x}$ & \\
\hline GeneSifter & $\mathrm{x}$ & $\mathrm{x}$ & $\mathrm{x}$ & $\mathrm{x}$ & $\mathrm{x}$ & $\mathrm{x}$ & $\mathrm{x}$ & \\
\hline
\end{tabular}

$\mathrm{x}=$ the software system addresses that specific aspect of the microarray process.

\section{DATA MANAGEMENT SYSTEMS}

These systems provide storage for, and management of, the massive quantities of data generated during the microarray process, usually including storage of the raw data files as well as sample and experimental annotation and analysis results.

\section{Biodiscovery's GeneDirector}

(http://biodiscovery.com/index/genedirector)

GeneDirector provides data management for information generated throughout the array experiment workflow. GeneDirector supports Windows, Linux, and Mac OSX, Oracle 8i, 9i.

\section{Stratagene's GeneTraffic (http://www.stratagene.com)}

GeneTraffic Software is a client-server-based microarray data management and analysis software package intended primarily for core facilities. GeneTraffic is available in two configurations, UNO for Affymetrix GeneChip data and DUO for two-color arrays, and provides data management for raw images as well as expression data and sample annotation. It also provides an Application Programming Interface (API) to integrate data with other software applications. It requires Linux server and Windows PC for client.

\section{PATHWAY/FUNCTIONAL/NETWORK ANALYSIS SYSTEMS}

These systems perform the task of building and visualizing the potential interactions between gene products leveraging databases of published literature. This type of system relies on databases generated by either automated extraction of the biological literature (text mining), usually of PubMed abstracts, or expert curation of the liter- ature to identify potential relationships between gene products, allowing users to uncover novel interactions between genes identified as being differentially expressed by their microarray research.

\section{Ingenuity Pathways Analysis (IPA) (http://www.ingenuity.com/)}

Ingenuity Pathways Analysis is a web-based application that allows users to analyze pathway information from Ingenuity's extensive knowledge base. The user can use this curated data to put their microarray results in the context of biological pathways and also to predict interactions or gene networks.

\section{PubGene (http://www.pubgene.com)}

PubGene provides up-to-date information on gene and protein relationships from the literature that can be used to put microarray results in the context of possible new interactions or gene networks. Web-based application provides secure web access from any computer platform.

\section{Ariadne Pathway Studio (formerly known as Pathway Assist) \\ (http://www.ariadnegenomics.com/products/pathway/)}

Pathway Studio is pathway analysis software for interpreting experimental results in the context of pathways, gene regulation networks, and protein interaction maps. Pathway Studio is a desktop software application compatible with Windows computers.

\section{DATA ANALYSIS/INTEGRATED SYSTEMS}

These systems provide analysis methods for identification of differentially expressed genes as well as methods for data visualization and partitioning. Most systems also address biological significance by providing access to gene annotation and functional/pathway analysis. 
Insightful's S+ArrayAnalyzer

(http://www.insightful.com/industry/pharm/)

ArrayAnalyzer includes methods for data access to internal databases as well as analysis and annotation features. S+ArrayAnalyzer is extendable using S-PLUS scripts and well-documented $\mathrm{C}++$, Java, SOAP, JavaServer Pages Tag APIs. S+ArrayAnalyzer runs on Windows, Linux, and Solaris and includes web (server) and desktop deployment options.

\section{Biodiscovery's GeneSight (http://biodiscovery.com/index/genesight)}

GeneSight provides visualization and statistical decision support tools. The Integrated Annotation Complier supports Affymetrix annotation files and annotations from other public databases. GeneSight is a desktop software application compatible with Windows, Linux, and Mac OS X.

\section{Agilent GeneSpring (http://www.genespring.com)}

GeneSpring is a desktop software application with macros for writing specialized bioinformatics scripts. Programmers can use GeneSpring's Application Programming Interface (API) to extend functionality. The API enables incorporation of third-party applications such as BioConductor, SAS, MATLAB, and R algorithms. GeneSpring is a desktop software application compatible with Windows and Mac OS X.

\section{Informax Vector Xpression}

\section{(http://www2.informaxinc.com/solutions/xpression/} main.html)

Vector Xpression provides visualization and statistical analysis tools and external links to external web applications and databases of choice for gene annotation. Vector Xpression is a desktop software application compatible with Windows.

\section{Partek Genomics Suite}

(http://www.partek.com/html/products/products.html)

Partek's Genomics Suite provides integrated statistical and visual analysis and is optimized for fast and memory-efficient processing of large, ultra-high-dimensional data. Genomics Suite provides a large selection of class prediction methods for diagnostic/prognostic prediction. Partek GS provides automatic annotation of results and provides hyperlinks to Affymetrix NetAffx annotation and other databases.

\section{Rosetta Biosoftware's Rosetta Resolver System (http://www.rosettabio.com/products/resolver/ default.htm)}

The Rosetta Resolver system is an enterprise-wide system gene expression analysis that combines analysis tools with a robust, scalable database designed primarily for pharmaceutical and biotechnology companies.
Spotfire's DecisionSite for Microarray Analysis (http://spotfire.com/products/decisionsite microarray_analysis.cfm)

DecisionSite for Microarray Analysis provides visual analytics with statistics for analysis of both Affymetrix GeneChip and 2-Channel microarray experiments. The application can be extended to interactively incorporate custom statistical methods written in other packages such as SAS, Insightful, SciTegic, or R.

\section{Stratagene's ArrayAssist \\ (http://www.stratagene.com)}

ArrayAssist is a desktop software application for the analysis of Affymetrix GeneChip expression data. ArrayAssist can perform statistical analysis with $p$ value corrections, cluster analysis, and data visualization and provides access to Affymetrix NetAffx annotation. ArrayAssist is compatible with Microsoft Windows 2000 and Windows XP operating systems.

\section{VizX Labs' GeneSifter (http://www.genesifter.net)}

GeneSifter supports Affymetrix GeneChip, GE Healthcare CodeLink arrays, and Illumina arrays as well as both commercial and custom two-color arrays. GeneSifter provides comparison statistics, correction for multiple testing, and cluster analysis with secure web access from any computer platform. Automatic updates of gene annotation and integrated tools for summarizing function and pathway information associated with a gene list are provided.

\section{CONCLUSION}

The microarray data analysis process continues to be a challenge for many biomedical researchers using microarray technology to examine gene expression on a global scale. One of the main causes of challenge has been the software available for this process. Proper experimental design and the use of an adequate number of replicates will make the identification of differentially expressed genes straightforward with the use of wellestablished statistical tests. However, the researcher must be able to implement the tests and, even if this is accomplished, the analysis process does not end with the identification of differentially expressed genes. From the biologist's perspective, in many ways the real work begins with the struggle to make biological sense of gene lists often containing thousands of genes. For researchers working with human, mouse, or rat microarrays or other well-annotated model organisms, the struggle is not due to a lack of information for the identified genes but rather the magnitude of the task of collecting and examining gene information from multiple databases for thousands of genes. Despite the availability of software for both the identification of differentially expressed genes and tools for determining the biological significance of these 
genes, the analysis challenge continues quite often because of issues such as a mismatch between the analysis methods needed for a specific experimental design and those available in the software at hand, difficulty in accessing a single user desktop system when needed, or a failure to make use of an analysis system due to a lack of training needed to use the system. One of the most important and most often overlooked aspects is accessibility; if a researcher cannot use the functions available in a system, success will be evasive regardless of how rich the choices. This review has tried to address some of the aspects of the analysis that may be limiting analysis success. Taking all of these aspects into account, in addition to aspects such as statistical analysis when planning microarray experiments, including the analysis process will greatly increase the chances of success in the analysis process.

Researchers have many diverse software options available for microarray data analysis including both noncommercial and commercial options. The statistical analysis needs as well as the requirements for addressing biological significance can be met by all or some combination of all of the options presented in this review; therefore, the researcher can include the aspects of the analysis process presented here in their decision-making process. For many biomedical researchers commercial systems will be the best choice. While noncommercial options can be used and may offer many advantages for researchers with bioinformatics skills or access to those skills, commercial software offers an attractive alternative to those researchers who lack these skills and are not interested in developing them.

\section{REFERENCES}

1. Miller RM, Federoff HJ. Microarrays in Parkinson's Disease: A Systematic Approach. NeuroRx 3:318-325, 2006.

2. Miller LD, Long PM, Wong L, Mukherjee S, McShane LM, Liu ET. Optimal gene expression analysis by microarrays. Cancer Cell 2:353-361, 2002.

3. Draghici S. Statistical intelligence: effective analysis of high-density microarray data. Drug Discovery Today 7:S55-S63, 2002.

4. Pavlidis P. Using ANOVA for gene selection from microarray studies of the nervous system. Methods 31:282-289, 2003.

5. Pavlidis P, Li Q, Noble WS. The effect of replication on gene expression microarray experiments. Bioinformatics 19:16201627, 2003

6. Borovecki F, Lovrecic L, Zhou J, Jeong H, Then F, Rosas HD, et al. Genome-wide expression profiling of human blood reveals biomarkers for Huntington's disease. Proc Natl Acad Sci USA 102: 11023-11028, 2005.

7. Barnes M, Freudenberg J, Thompson S, Aronow B, Pavlidis P. Experimental comparison and cross-validation of the Affymetrix and Illumina gene expression analysis platforms. Nucleic Acids Res 33:5914-5923, 2005.

8. Larkin JE, Frank BC, Gavras H, Sultana R, Quackenbush J. Independence and reproducibility across microarray platforms. Nat Methods 2:337-344, 2005.
9. Irizarry RA, Warren D, Spencer F, Kim IF, Biswal S, Frank BC, et al. Multiple-laboratory comparison of microarray platforms. Nat Methods 2:345-350, 2005.

10. Reimers M, Heilig M, Sommer WH. Gene discovery in neuropharmacological and behavioral studies using Affymetrix microarray data. Methods 37:219-228, 2005.

11. Bolstad BM, Irizarry RA, Astrand M, Speed TP. A comparison of normalization methods for high density oligonucleotide array data based on variance and bias. Bioinformatics 19:185-193, 2003.

12. Quackenbush J. Microarray data normalization and transformation. Nat Genet 32:S496-S501, 2002.

13. Rosati B, Grau F, Kuehler A, Rodriguez S, McKinnon D. Comparison of different probe-level analysis techniques for oligonucleotide microarrays. Biotechniques 36:316-322, 2004.

14. Wu W, Dave N, Tseng GC, Richards T, Xing EP, Kaminski N. Comparison of normalization methods for CodeLink Bioarray data. BMC Bioinformatics 6:309, 2005.

15. Almudevar et al. NeuroRx 2006.

16. Baldi P, Long AD. A Bayesian framework for the analysis of microarray expression data: regularized $t$-test and statistical inferences of gene changes. Bioinformatics 17:509-519, 2001.

17. Benjamini Y, Hochberg Y. Controlling the false discovery rate: a practical and powerful approach to multiple testing. J R Stat Soc B 57, 289-300, 1995.

18. Dudoit S, Shaffer JP, Boldrick JC. Multiple hypothesis testing in microarray experiments. Stat Sci 18:71-103, 2003.

19. Reiner A, Yekutieli D, Benjamini Y. Identifying differentially expressed genes using false discovery rate controlling procedures. Bioinformatics 19:368-375, 2003.

20. Kaufman L, Rousseeuw PJ. Finding groups in data: an introduction to cluster analysis. New York: Wiley; 1990.

21. Eisen MB, Spellman PT, Brown PO, Botstein D. Cluster analysis and display of genome-wide expression patterns. Proc Natl Acad Sci USA 95:14863-14868, 1998.

22. Quackenbush J. Computational analysis of microarray data. Nat Rev Genet 2:418-427, 2001.

23. Wheeler DL, Barrett T, Benson DA, Bryant SH, Canese K, Chetvernin V, et al. Database resources of the National Center for Biotechnology Information. Nucleic Acids Res 34:D173-D180, 2006.

24. Doniger SW, Salomonis N, Dahlquist KD, Vranizan K, Lawlor SC, Conklin BR. MAPPFinder: using Gene Ontology and GenMAPP to create a global gene-expression profile from microarray data. Genome Biol 4:R7, 2003.

25. Pavlidis P, Qin J, Arango V, Mann JJ, Sibille E. Using the gene ontology for microarray data mining: a comparison of methods and application to age effects in human prefrontal cortex. Neurochem Res 29:1213-1222, 2004.

26. Ball CA, Sherlock G, Parkinson H, Rocca-Sera P, Brooksbank C, Causton HC, et al. Microarray Gen Expression Data (MGED) Society. Standards for microarray data Science 298:539, 2003.

27. Brazma A, Hingamp P, Quackenbush J, Sherlock G, Spellman P, Stoeckert C, et al. Minimum information about a microarray experiment (MIAME): toward standards for microarray data. Nat Genet 29:365-371, 2001.

28. MIAME checklist. The Microarray Gene Expression Data (MGED) Society. Available at: http://www.mged.org/ Workgroups/MIAME/miame_checklist.html. Accessed: June 5, 2006.

29. Barrett T, Suzek TO, Troup DB, Wilhite SE, Ngau WC, Ledoux P, et al. NCBI GEO: mining millions of expression profiles. Database and tools. Nucleic Acids Res 33:D562-D566, 2005.

30. Knudsen S. A biologist's guide to analysis of DNA microarray data. New York: Wiley-Liss, 2002.

31. Parmigiani G, Garret E, Irizarry R, Zeger S. The analysis of gene expression data: methods and software. New York: Springer; 2003.

32. Quackenbush J. Open-source software accelerates bioinformatics. Genome Biol 4:336, 2003.

33. Dudoit S, Gentleman RC, Quackenbush J. Open source software for the analysis of microarray data. Biotechniques S45-S51, 2003. 